This is the author's final, peer-reviewed manuscript as accepted for publication. The publisher-formatted version may be available through the publisher's web site or your institution's library.

\title{
Analysis of optimal reconfiguration of shipboard power systems
}

Sayak Bose, Siddharth Pal, Balasubramaniam Natarajan, Caterina M. Scoglio, Sanjoy Das, and Noel N. Schulz

\section{How to cite this manuscript}

If you make reference to this version of the manuscript, use the following information:

Bose, S., Pal, S., Natarajan, B., Scoglio, C. M., Das, S., \& Schulz, N. N. (2012). Analysis of optimal reconfiguration of shipboard power systems. Retrieved from http://krex.ksu.edu

\section{Published Version Information}

Citation: Bose, S., Pal, S., Natarajan, B., Scoglio, C. M., Das, S., \& Schulz, N. N. (2012). Analysis of optimal reconfiguration of shipboard power systems. IEEE Transactions on Power Systems, 27(1), 189-197.

Copyright: (C) 2011 IEEE

Digital Object Identifier (DOI): doi:10.1109/TPWRS.2011.2163948

Publisher's Link: http://ieeexplore.ieee.org/xpl/articleDetails.jsp?arnumber=6009217

This item was retrieved from the K-State Research Exchange (K-REx), the institutional repository of Kansas State University. K-REx is available at http://krex.ksu.edu 


\title{
Analysis of Optimal Reconfiguration of Shipboard Power Systems
}

\author{
Sayak Bose, Student Member, IEEE, Siddharth Pal, Balasubramaniam Natarajan, Senior Member, IEEE, \\ Caterina M. Scoglio, Member, IEEE, Sanjoy Das, and Noel N. Schulz, Senior Member, IEEE
}

\begin{abstract}
In power system reconfiguration, the status (ON/OFF) of switches are optimized such that maximum power is delivered to loads after the occurrence of a fault. The optimized reconfiguration is achieved by prioritizing power delivered to vital loads over semi-vital and nonvital loads. The formulation presented in this paper considers a new balanced hybrid (AC and DC) shipboard power system (SPS). Analysis of the nonconvex reconfiguration formulation is done by an appropriate nonconvex solver and by convex approximation. Unlike the nonconvex solution that is based on branch-and-bound methods, convex approximation significantly reduces complexity. It is shown that for the hybrid SPS reconfiguration problem, low complexity convex approximations are effective in finding optimal solutions. Cumulative distribution function (CDF) of the power delivered to loads is presented to showcase the system robustness against random fault scenarios. A combined objective of maximizing power delivery and minimizing the number of switching actions is included in the analysis. Tradeoff between power delivered and number of switching operations after reconfiguration has been discussed at steady state. A separate analysis is also included to observe the intermediate dynamic switch states while the reconfiguration is in progress to capture the tradeoff more prominently.
\end{abstract}

Index Terms-Optimization, reconfiguration, shipboard power systems.

\section{INTRODUCTION}

C HIPBOARD power systems essentially need automated reS configuration to maintain continued service to loads after a fault. It is often proved that an integrated power system (IPS) provides a better solution than the manual process of restoration in terms of fight-through and survivability. In this process, multiple generators of various sizes and power generation capabilities are placed throughout the ship. IPS minimizes the amount of service interruption to affected portion of the ship during battle damage or any other sudden faults.

Traditionally, reconfiguration for service restoration for terrestrial systems has been observed as an optimization problem

Manuscript received October 07, 2010. revised October 21, 2010, October 22, 2010, May 21, 2011, and July 20, 2011; accepted July 27, 2011. This work was supported by the United States Office of Naval Research under grant N00014-10-1-0431 (DEPSCoR program). Paper no. TPWRS-00804-2010.

$\mathrm{S}$. Bose is with the Department of Electrical and Computer Engineering, Kansas State University, Manhattan, KS 66506 USA (e-mail: sayak@k-state. edu).

S. Pal was with Kansas State University, Manhattan, KS 66506 USA, and is now with Jadavpur University, Kolkata, India (e-mail: sidd_pa12002@yahoo. com).

B. Natarajan, C. M. Scoglio, S. Das, and N. N. Schulz are with Kansas State University, Manhattan, KS 66506 USA (e-mail: bala@ksu.edu; caterina@kstate.edu; sdas@k-state.edu; noels@k-state.edu).

Digital Object Identifier 10.1109/TPWRS.2011.2163948 that uses the objective as the sum of power or current delivered to the loads [1]. Mixed integer nonlinear programming techniques were employed to solve the problem. Other reconfiguration objectives, such as power loss reduction [2] and stability margins [3], have been considered. Heuristic approaches [4], expert systems [5] based strategies, and mathematical programming [6] approaches have also been proposed. In addition, a combination of genetic algorithm and fuzzy logic [7] is used to solve large terrestrial systems reconfiguration problem. However, these efforts typically require running a complete power flow algorithm after each switching step (to determine if the constraints are satisfied), making the process slow and infeasible in some cases. Hence, better approaches are based on optimizing the objective function while simultaneously satisfying the power flow constraints.

A shipboard power system (SPS) is nonlinear and several methods using optimization for SPS have been proposed. Butler et al. [8] first proposed a novel fixed charge network flow method for reconfiguration for restoration based on maximizing the power delivered to loads while satisfying radiality constraints. The optimization was further improved to satisfy more constraints [9], including heuristic methods [10], and incorporate geographic fault information [11]. Other extensions of these methodologies account for nonradial topologies and mixed AC/DC systems [12], as well as islanding scenarios [13]. For these methods, however, bi-directional flow of current was not considered which is inadequate for multiple fault scenarios and distributed generators (DG). The formulation for the reconfiguration problem considering both DG and bi-directional power flow results in a nondeterministic polynomial-time (NP) hard problem and was first presented in [14]. A global optimum to this problem can be found by branch-and-bound [15] methods or exhaustive search.

First, this paper, with the aid of a new MVDC ship model, demonstrates that low-complexity methods can be effectively applied to the reconfiguration problem to produce solutions that are near-optimal. With reduction in complexity, near-optimal solution can be reached in milliseconds, which makes it an attractive option to be used with future real-time SPSs. In [16], an analytical view of the methods to solve the reconfiguration problem is presented for the first time in the literature, and are applied to solve the reconfiguration problem for the new MVDC ship model in this paper. Fig. 1 summarizes the approaches. With a new notional shipboard model in [17], the effectiveness of the solution approaches is demonstrated. To accomplish this, the same process as described in [16] is followed. Specifically, the branch-and-bound based exhaustive search is applied to the 


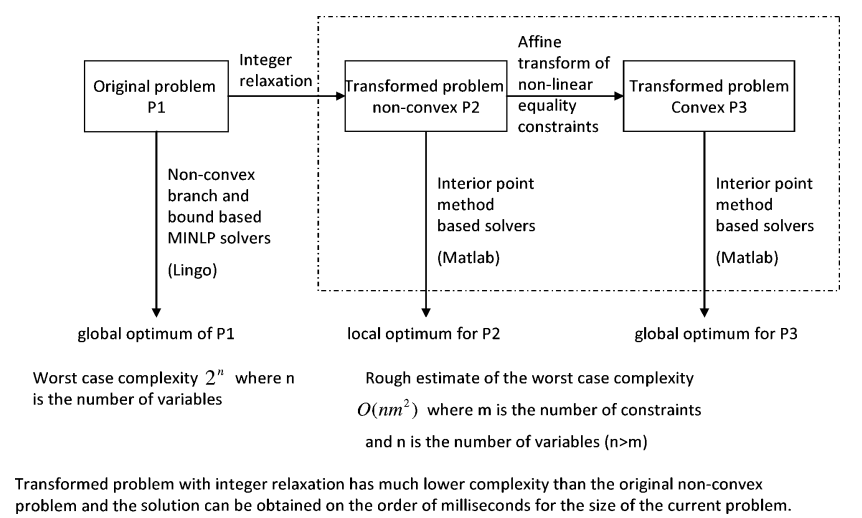

Fig. 1. Methods to analyze optimal solutions to SPS reconfiguration problem.

original nonconvex mixed integer problem $P 1$ to find a global optimum switch configuration. Next, the original problem $P 1$ is converted to a continuous nonconvex problem $P 2$ by relaxing the integer constraints on the switches such that $X \in[0,1]$ where $X$ is any switch variable. Interior-point based methods [18] are applied to find the local optimum solution. Steady-state simulation analysis indicates that the local optimum obtained from integer relaxation followed by rounding matches closely with the global optimum found by the branch and bound. Next, the nonconvex relaxed integer problem $P 2$ is converted to a convex relaxed integer problem $P 3$ through affine transformation of the equality constraints. The global solution to this transformed convex problem is found by applying an interior-point method based solver. Extensive simulation with various fault cases reveal that the global optimum for $P 3$ and the local optimum for $P 2$ closely match with the optimal reconfiguration solution of the original nonconvex mixed integer problem $P 1$ with high regularity. This may be possible as the nonconvexity of the original problem is in fact limited to a few nonlinear equality constraints in the $\mathrm{AC}$ power flow section of the generator while the majority of the formulation is convex.

Second, robustness of the system is analyzed by considering the cumulative distribution of the power delivered in the event of $K$ random fault cases (followed by reconfiguration). In this case, all vital and semi-vital loads are served partially or fully within a certain probability. This approach aims at quantifying the system robustness against faults as in [17], but adds more information on power delivery to each category of loads under a fault scenario.

Finally, the tradeoff between power delivered and the number of switching operations needed to restore power is captured. Specifically, a bi-objective optimization problem is formulated with a second objective of minimizing the number of switching operations and "scalarize" it with the first objective of maximizing power delivered to loads. Empirical solutions of this combined weighted dual objective is presented against a random 2 -fault case. The analysis is easily extendable to any $K$ random fault cases.

This paper is organized as follows: The shipboard power system model is discussed in Section II. A detailed description of the reconfiguration methods is provided in Section III. Simulation of reconfiguration results on a new notional shipboard model [17] and further analysis with the present model in

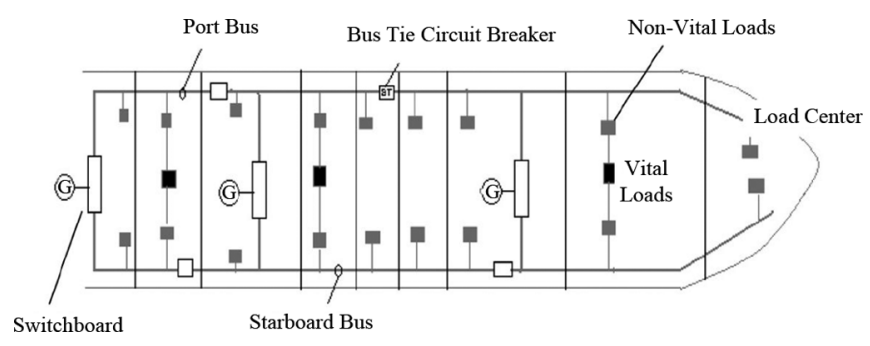

Fig. 2. Zonal architecture of shipboard power system (from [19]).

Fig. 2 are presented in Section IV, Computational complexity of the original and proposed methods for reconfiguration are discussed in Section V, and finally, conclusions are summarized in Section VI.

\section{OVERVIEW OF SHIPBoARd Power System Model}

Traditionally, SPS systems are self-contained and have radial distribution architectures. The space and weight constraints limit the amount of redundancy that can be incorporated into the system for reconfiguration and restoration purposes. The SPS distribution network is tightly coupled, and therefore, resistive losses are negligible. Recently, some researchers have proposed a radial distribution architecture with a zonal approach that employs a starboard bus (SB) and a port bus (PB), and thus partitioning the ship into a number of electric zones [19]. For our model, as shown in Fig. 2, a DC zone electric distribution system is considered. The power from the output of the generators is first converted into DC and fed into the loads, which may need reconversion to AC. Each zone has two load centers driven by generator switchboards radially from PB and SB. Three kinds of loads are considered in this model: vital loads, semi-vital loads that are required for combat operations, and nonvital loads. It is assumed that nonvital loads can be shed to maintain power in the vital and semi-vital loads in case of emergencies. Firstly, the reconfiguration methods presented in [16] are reapplied on a new notional SPS model [17] to show the effectiveness of the low-complexity methods. This new model has four generators and seven load centers. Load priorities and other constraints similar to the model in Fig. 2 are imposed on the new model which are discussed in detail in Section III. Further analysis in this paper, related to robustness to faults, is done on the model in Fig. 2 (an extension of the analysis already presented in [16]).

\section{RECONFIGURATION FORMULATION}

In this section, three formulations for SPS reconfiguration are presented. They include the original mixed-integer nonconvex (MINLP), relaxed-integer nonconvex, and relaxed integer convex formulations. A description for each is provided in the following subsections. Also a dual objective formulation is included that attempts to maximize power delivery to loads while minimizing number of switching operations.

\section{A. Mixed-Integer Nonconvex Formulation (P1)}

In this formulation, the objective function and the constraints are similar to the ones presented in [14] and [16]. However, this paper primarily extends the work in [16] to include examples 
of new balanced SPS systems. Once again, we are interested in determining the optimal switch configuration that maximizes the power delivered to loads. The detailed formulations are presented below: Objective function:

$$
\operatorname{Max} \sum_{I \in L} W_{V L}^{\prime} S_{V L_{I}}^{\prime}+W_{S V L_{I}}^{\prime} S_{S V L_{I}}^{\prime}+W_{N V L_{I}}^{\prime} S_{N V L_{I}}^{\prime}
$$

Subject to AC constraints Equality constraints:

$$
\begin{aligned}
& P G_{i}-P D_{i}=\sum_{i} V_{i} V_{j} V_{i j} \operatorname{Re}\left\{\angle\left(\theta_{i j}+\delta_{j}-\delta_{i}\right)\right\} \\
& Q G_{i}-Q D_{i}=\sum_{i} V_{i} V_{j} V_{i j} \operatorname{Im}\left\{\angle\left(\theta_{i j}+\delta_{j}-\delta_{i}\right)\right\}
\end{aligned}
$$

Inequality constraints:

$$
\begin{aligned}
P G_{i}^{\min } & \leq P G_{i} \leq P G_{i}^{\max } \\
Q G_{i}^{\min } & \leq Q G_{i} \leq Q G^{\max } \\
I_{i j} & \leq I_{i j}^{\max } \\
V_{i}^{\min } & \leq V_{i} \leq V_{i}^{\max } \\
\delta_{i}^{\min } & \leq \delta_{i} \leq \delta_{i}^{\max }
\end{aligned}
$$

DC constraints Equality constraints:

$$
\begin{aligned}
\sum_{i} I_{i n_{i}} & =\sum_{i} I_{\text {out }_{i}}+I L_{i} i \in F B, j \in T B \\
V_{i} & =V_{j}+I_{i j} \times Z_{i j}
\end{aligned}
$$

Inequality constraints:

$$
\begin{aligned}
P L_{i} & \leq P L_{i}^{\max } \times S W_{i} \text { for variable load } \\
P L_{i} & =B_{i} \times P L_{i}^{\max } \times S W_{i} \text { for fixed load } \\
I_{i j} & \leq I_{i j}^{\max } \\
V_{i}^{\min } & \leq V_{i} \leq V_{i}^{\max }
\end{aligned}
$$

Switching constraints:

$$
S W_{i}+S W_{j}=1 \text { where } S W \in\{0,1\}
$$

Several important observations can be drawn from (1)-(15) which are discussed in the following.

1) Weight are associated to prioritize service to different types of load as discussed in I. We assign $W_{N V L}^{\prime}=1$ and assume that $W_{V L}^{\prime}>W_{S V L}^{\prime}>W_{N V L}^{\prime}$ where $N V L, V L$, and $S V L$ are nonvital, vital, and semi-vital loads, respectively. Specifically, $W_{V L}^{\prime}=100$ and $W_{S V L}^{\prime}=10$ are picked for our analysis. $L$ represents the set of loads in the power system.

2) Several constraints involving power flow, generator power limits, load limits, bus current, and voltage limits are imposed. $P G$ and $Q G$ are the active and reactive power, respectively, generated from the $\mathrm{AC}$ generator. $P D$ and $Q D$ are the active and reactive power demanded, $V_{i}$ is the voltage at bus $i, \delta_{i}$ is the angle associated with the voltage at bus $i, Y_{i j}$ is the magnitude of the complex admittance from bus $i$ to $j, \theta_{i j}$ is the angle of the admittance from bus $i$ to $j, P L_{i}$ is the power delivered to loads connected at bus $i$, and $B_{i}$ is a binary variable that connects a fixed load to
PB or SB and can be predetermined. $I L_{i}$ is the load current at bus $i, I_{i j}$ is the current flow from bus $i$ to bus $j$, $I_{i n_{i}}$ and $I_{\text {out }_{i}}$ are the currents entering and leaving bus $i$, $F B$ is the set of "from (source)" buses, $T B$ is the set of "To (destination)" buses, and $Z_{i j}$ is the branch impedence of branch $i$ and $j .(.)^{\max }$ and (.) ${ }^{\mathrm{min}}$ are used to indicate the maximum and minimum value of each variable, respectively. Unless explicitly mentioned, all variables indicate their magnitudes.

3) Switches are formulated as binary variables. The mutual exclusivity constraints on the switches determine if the power delivered to the higher priority loads is from port side or starboard side.

\section{B. Relaxed-Integer Non-Convex Formulation (P2)}

In order to reduce complexity of the original MINLP problem, the switches are relaxed to have any value between 0 and 1, i.e., $S W \in[0,1]$, while the mutual exclusivity constraint shown in (15) still holds good. This problem can be solved by interior point based nonlinear solvers.

\section{Relaxed-integer Convex Formulation (P3)}

A convex form of the previous relaxed integer formulation is obtained by affine transformation of the nonlinear equality constraints using Newton's power flow method [20]. In this, the power flow (2) and (3) are rewritten as follows:

$$
\begin{aligned}
P G_{i}-P D_{i}= & V_{i} \sum_{j \neq i} V_{j} Y_{i j} \cos \left(\theta_{i j}+\delta_{i}-\delta_{j}\right) \\
& +V_{i}^{2} Y_{i j} \cos \left(\theta_{i j}\right) \\
Q G_{i}-Q D_{i}= & V_{i} \sum_{j \neq i} V_{j} Y_{i j} \sin \left(\theta_{i j}+\delta_{i}-\delta_{j}\right) \\
& +V_{i}^{2} Y_{i j} \cos \left(\theta_{i j}\right) .
\end{aligned}
$$

Using (16) and (17), the Jacobian $\mathcal{J}$ is calculated and the incremental change in voltages and angles at every step of iteration from initial values of the angles $\delta_{i}$ and voltages $V_{i}$ is obtained. So the constraints take linear forms as

$$
\left[\begin{array}{c}
\Delta P G_{i} \\
\Delta Q G_{i}
\end{array}\right]=\left[\mathcal{J}_{i}\right]\left[\begin{array}{l}
\Delta \delta_{i} \\
\Delta V_{i}
\end{array}\right]
$$

It is easy to show that, for a finite range of $\delta_{i}$ and $V_{i}$, the constraints given in (18) are affine. Therefore, this problem is a relaxed-integer convex problem and the global optimum for this problem can be found using an interior point method based solver.

It is shown subsequently that the optimal solutions provided by the low-complexity solvers discussed above provide close match with the global solution. This is a crucial feature for a "good quality" sub-optimal solution in general.

\section{Dual-Objective Formulation}

Another objective of minimizing the number of switching operations while maximizing the power delivered to the loads is now introduced. The purpose of introducing this objective is to provide a trade-off between power delivered and switching operations performed as each switching operation incurs power loss and could result in undesirable transients in 
the system. To describe the formulation, first, the minimum "Hamming" distance from pre-fault switch state to the current switch state is taken into account. This distance is given as: $T(X)=S W_{\text {prefault }_{i}}-S W_{i}$, where $i \in 1, \ldots, N$. With MINLP formulation, the two objective functions are given as follows:

$$
\begin{aligned}
F_{1}= & \operatorname{Max} \sum_{I \in L} W_{V L}^{\prime} S_{V L_{I}}^{\prime} 1 \\
& +W_{S V L_{I}}^{\prime} S_{S V L_{I}}^{\prime}+W_{N V L_{I}}^{\prime} S_{N V L_{I}}^{\prime} \\
F_{2}= & \operatorname{Min} \sum_{N}^{X=1} T(X) .
\end{aligned}
$$

With the relaxed-integer cases, the "Hamming" distance in (20) is changed to the "Euclidian" distance which is given by $E(X)=\left(S W_{\text {prefault }_{i}}-S W_{i}\right)^{2}$ so that it becomes

$$
F_{2}=\operatorname{Min} \sum_{N}^{X=1} E(X) .
$$

The optimization problem defined above has two objective functions, $F_{1}$ and $F_{2}$, that work against each other. That is, as each switching operation in $F_{1}$ attempts to increase power in order to maximize power delivered, the constraints in (2)-(15) become difficult to satisfy unless more switching operations are performed. Therefore, $F_{2}$ will increase if $F_{1}$ is increased and vice versa. The domains of objective $F_{1}$ and $F_{2}$ are the same, and in particular are convex, as they both are functions of switch variables. The multi-objective domain is therefore convex. It is common to combine such mutually conflicting objectives into a single objective function using the "weighted sum" approach [21] and look at pareto optimal solutions. The combined single objective optimization problem can be formulated as follows:

$$
F=w \times F_{1}-(1-w) \times F_{2} .
$$

The parameters $w$ and $(1-w)$ in the combined objective function reflect "importance" of the corresponding objective function and may vary from 0 to 1 . If the two functions have comparable values, the $w$ parameter in (22) can meaningfully capture the trade-off between $F_{1}$ and $F_{2}$. In this case, since $F_{1}$ is a function of power and $F_{2}$ depends on switching operations, different ranges for $F_{1}$ and $F_{2}$ are possible. Therefore, in order to bring $F_{1}$ and $F_{2}$ to a comparable scale, the "upperlower-bound" transformation technique suggested for multi-objective optimization problem in [22] is used. In this approach, the transformed objectives $F_{1}^{t f}$ and $F_{2}^{t f}$ are obtained such that the weighted dual objective becomes

$$
F=w \times F_{1}^{t f}-(1-w) \times F_{2}^{t f}
$$

subject to constraints (2)-(15). $F_{1}^{t f}$ and $F_{2}^{t f}$, formulated as functions of the optimization variable $x$ are as follows:

$$
F_{n}^{t f}(x)=\frac{F_{n}(x)-F_{n}^{0}(x)}{F_{n}^{\max }(x)-F_{n}^{0}(x)}, n=1,2 .
$$

$F_{n}^{\max }(x)$ and $F_{n}^{0}(x)$ are given by

$$
F_{n}^{\max }(x)=\underset{\leq l \leq n}{\operatorname{Max}} F_{n}\left(x_{l}^{*}\right), n=1,2
$$

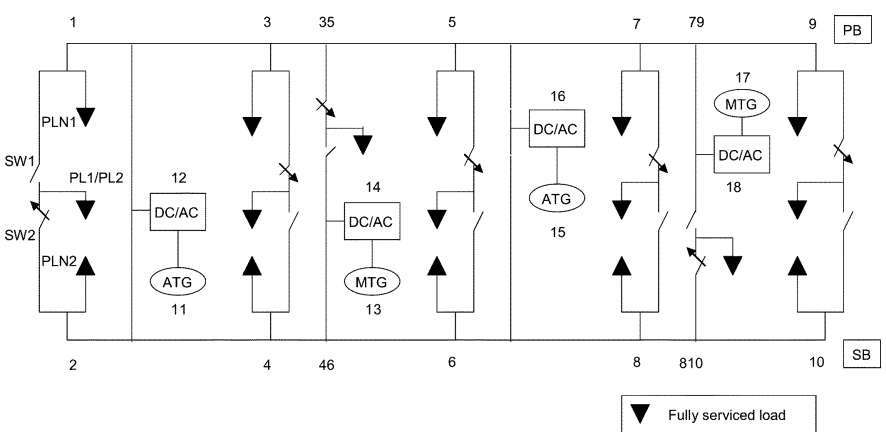

Fig. 3. Schematic view of SPS under normal condition (pre-fault).

$$
F_{n}^{0}(x)={ }_{x}^{\operatorname{Min}}\left(F_{n}(x) \mid x \subset X\right), n=1,2 .
$$

Here, $X$ is the design space, $x_{l}^{*}$ is the point that maximizes the first objective function and $n$ is the number of objective functions. With this transformation, $F_{1}^{t f}$ and $F_{2}^{t f}$ now typically range between 0 and 1 .

\section{ANALYSIS OF RESULTS}

In this section, first, solutions to the reconfiguration problem from the three different formulations are compared. The original nonconvex reconfiguration problem is solved using "LINGO" software package, while the other two formulations are solved using interior point methods in MATLAB. Second, a power flow $\mathrm{CDF}$ is presented to analyze the robustness of the system under study, and finally, the tradeoff between power delivered and number of switching operations is analyzed based on the dual objective formulation. To obtain the power flow CDF and to analyze the tradeoff, the transformed relaxed-integer convex formulation is used.

A new MVDC ship model presented in [17] is used for validation and analysis of the formulations $P 1, P 2$, and $P 3$. Seven DC load zones are fed power from two main generators (MTG) and two auxiliary generators (ATG), with each MTG of $6 \mathrm{MW}$ and ATG of 2 MW generation capacities. Five load zones have vital, semi-vital, and nonvital loads while two load zones have vital loads only. Asynchronous bus transfer (ABT) is used to switch between $P B$ and $S B$, and is characterized by mutually exclusive switches as shown in Fig. 3. Another well-known notional ship model depicted in Fig. 2 is used for the rest of this analytical study. This model has seven load zones with two ship service converter module serving loads in each zone. A distributed generator (DG) along with the main generator $(\mathrm{G})$ is used to enhance the overall service capability. The main generator generates a maximum power of $16 \mathrm{MW}$ while the DG can generate up to $4 \mathrm{MW}$.

For each system discussed, it is assumed that 1) the vital loads require $0.5 \mathrm{MW}$, semi-vital loads require $1 \mathrm{MW}$, and nonvital loads require $0.5 \mathrm{MW}$ for operating in their respective full capacity. 2) The nonvital loads are directly serviced through the buses, while the vital and the semi-vital loads are serviced through the ABTs. 3) The combined power of the generators is sufficient to drive all the loads under normal operating condition. 4) All distributions are radial, as it provides advantages such as lower short-circuit current, easy switching, and less complex installation and functioning of sensor equipment. 


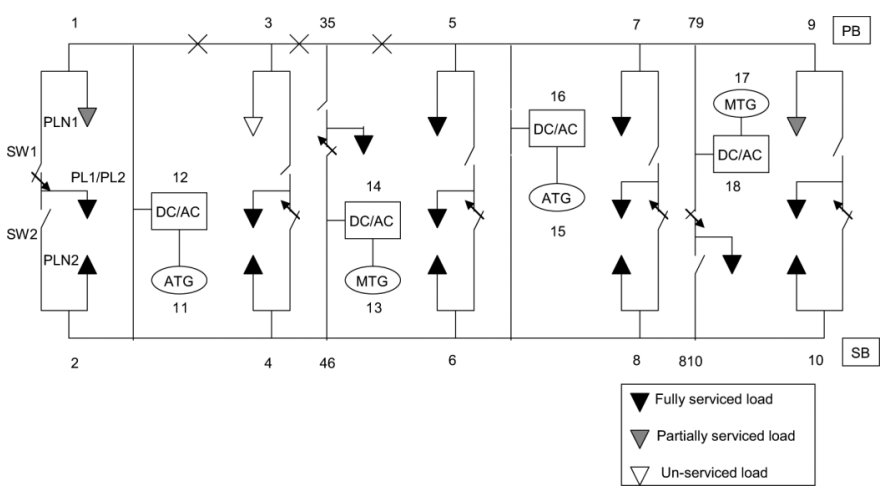

Fig. 4. Schematic view of SPS under faults occurring at $1-3,3-35$, and $35-5$.

The optimization methods described in Section III are applied to reconfigure the SPS after occurrence of one or more faults. Only steady-state reconfiguration status is considered in this paper. Table I shows the constrained parameters used in the simulations and their respective maximum and minimum values. First, an optimal pre-fault configuration for system model in [17] is presented, where all the loads are serviced to their full capacities. The total capacity of loads that are served under normal condition is $13.5 \mathrm{MW}$. It is assumed that, under steady-state, a component is unavailable whenever there is a fault; so the current through that component is forced to a very low value. This is achieved by increasing the branch resistance in the simulation. It is obvious that some loads are left without power after the fault. The reconfiguration formulations $P 1, P 2$, and $P 3$ ensure that the power is restored in a manner such that those loads are serviced optimally and according to their priority. This means that vital and semi-vital loads are restored before nonvital loads. Fig. 3 shows a pre-fault condition where all the loads are serviced for a particular switch configuration. Reconfiguration algorithms are applied for MINLP $(P 1)$, relaxed integer nonconvex $(P 2)$, and relaxed-integer convex $(P 3)$ cases from this initial (pre-fault) configuration. Now a fault scenario is considered where faults occur between branches $1-3,3-35$, and $35-5$. The portion of the PB between 1 and 5 is thus left without power and the switches need to change so that the loads can be serviced based to their priority mainly though the SB. The optimal reconfiguration, as it turns out in this case, requires the opening of $S W 2, S W 35, S W 5, S W 7, S W 810, S W 10$, and closing of $S W 1, S W 4, S W 6, S W 8, S W 79$, and $S W 9$ to ensure maximum power supply to the vital and semi-vital loads. The nonvital loads attached to the PB at nodes 1 and 9 are partially serviced due to insufficient power left in the generators, and the nonvital load attached to PB at node 3 is left unserviced. The power drawn from the main generators drops to 8.56 MW and the ATGs have to service to their full capacity. As seen from Table II, the optimal MINLP solver and near-optimal interior-point method based solvers produce the same switch status in this case. Fig. 4 also shows the switch status after reconfiguration.

Another fault scenario is considered, where four faults occur between branch $3-35,35-5,2-4$, and $6-8$. This creates an islanding scenario where loads on the left side of the
TABLE I

SiMULATION PARAMETERS

\begin{tabular}{|c|c|c|}
\hline Constrained parameters & max. & min. \\
\hline$P G_{i}$ (MTG) & $6 \mathrm{MW}$ & $0 \mathrm{MW}$ \\
\hline$P G_{i}(\mathrm{ATG})$ & $2 \mathrm{MW}$ & $0 \mathrm{MW}$ \\
\hline$I_{i j}$ & $500 \mathrm{~A}$ & $-500 \mathrm{~A}$ \\
\hline$V_{i}$ & $95 \mathrm{~V}$ & $105 \mathrm{~V}$ \\
\hline$\delta_{i}$ & $1^{\circ}$ & $-1^{\circ}$ \\
\hline$P L_{i}(\mathrm{VL})$ & $0.5 \mathrm{MW}$ & $0 \mathrm{MW}$ \\
\hline$P L_{i}(\mathrm{SVL})$ & $1 \mathrm{MW}$ & $0 \mathrm{MW}$ \\
\hline$P L_{i}(\mathrm{NVL})$ & $0.5 \mathrm{MW}$ & $0 \mathrm{MW}$ \\
\hline$S W_{i}$ & 1 & 0 \\
\hline$B_{i}$ & 1 & 0 \\
\hline
\end{tabular}

TABLE II

Power Delivered to LoAdS Under FaUlTS OCCURRING AT $1-3,3-35$, AND $35-5$

\begin{tabular}{|c|c|c|c|c|c|c|c|}
\hline $\begin{array}{l}\text { Load } \\
\text { positions }\end{array}$ & $\begin{array}{l}\text { Vital } \\
\text { (MW) }\end{array}$ & $\begin{array}{l}\text { Semi- } \\
\text { vital } \\
\text { (MW) }\end{array}$ & $\begin{array}{l}\text { Non- } \\
\text { vital } \\
\text { (MW) }\end{array}$ & $\begin{array}{l}\text { SW } \\
\text { positions } \\
\text { (closed) }\end{array}$ & $\begin{array}{l}\text { MTG } \\
\text { (Total) } \\
\text { (MW) }\end{array}$ & $\begin{array}{l}\text { ATG } \\
\text { (Total) } \\
\text { (MW) }\end{array}$ & Solver \\
\hline $\begin{array}{l}\text { Load } 1 / 2 \\
\text { Load } 3 / 4 \\
\text { Load } 35 \\
\text { Load 5/6 } \\
\text { Load } 7 / 8 \\
\text { Load } 810 \\
\text { Load } 9 / 10\end{array}$ & $\begin{array}{l}0.5000 \\
0.5000 \\
0.5000 \\
0.5000 \\
0.5000 \\
0.5000 \\
0.5000\end{array}$ & $\begin{array}{l}1.0000 \\
1.0000 \\
1.0000 \\
1.0000 \\
1.0000\end{array}$ & $\begin{array}{l}0.6319 \\
0.5000 \\
\\
1.0000 \\
1.0000 \\
0.6590\end{array}$ & $\begin{array}{l}\text { SW1 } \\
\text { SW4 } \\
\text { SW46 } \\
\text { SW6 } \\
\text { SW8 } \\
\text { SW810 } \\
\text { SW10 }\end{array}$ & 8.5590 & 4.0000 & $\begin{array}{l}\text { Global } \\
\text { Non-convex with } \\
\text { mixed integer } \\
\text { (LINGO) }\end{array}$ \\
\hline $\begin{array}{l}\text { Load } 1 / 2 \\
\text { Load 3/4 } \\
\text { Load } 35 \\
\text { Load 5/6 } \\
\text { Load } 7 / 8 \\
\text { Load } 810 \\
\text { Load 9/10 }\end{array}$ & $\begin{array}{l}0.5000 \\
0.5000 \\
0.5000 \\
0.5000 \\
0.5000 \\
0.5000 \\
0.5000\end{array}$ & $\begin{array}{l}1.0000 \\
1.0000 \\
1.0000 \\
1.0000 \\
1.0000\end{array}$ & $\begin{array}{l}0.6319 \\
0.5000 \\
1.0000 \\
1.0000 \\
\\
0.6590\end{array}$ & $\begin{array}{l}\text { SW1 } \\
\text { SW4 } \\
\text { SW46 } \\
\text { SW6 } \\
\text { SW8 } \\
\text { SW810 } \\
\text { SW10 }\end{array}$ & 8.5590 & 4.0000 & $\begin{array}{l}\text { Local } \\
\text { Non-convex with } \\
\text { integer relaxation } \\
\text { (MATLAB) }\end{array}$ \\
\hline $\begin{array}{l}\text { Load } 1 / 2 \\
\text { Load } 3 / 4 \\
\text { Load } 35 \\
\text { Load 5/6 } \\
\text { Load } 7 / 8 \\
\text { Load } 810 \\
\text { Load } 9 / 10\end{array}$ & $\begin{array}{l}0.5000 \\
0.5000 \\
0.5000 \\
0.5000 \\
0.5000 \\
0.5000 \\
0.5000\end{array}$ & $\begin{array}{l}1.0000 \\
1.0000 \\
1.0000 \\
1.0000\end{array}$ & $\begin{array}{l}0.6319 \\
0.5000 \\
\\
1.0000 \\
1.0000\end{array}$ & $\begin{array}{l}\text { SW1 } \\
\text { SW4 } \\
\text { SW46 } \\
\text { SW6 } \\
\text { SW8 } \\
\text { SW810 } \\
\text { SW10 }\end{array}$ & 8.5590 & 4.0000 & $\begin{array}{l}\text { Global } \\
\text { Convex with } \\
\text { integer relaxation } \\
\text { (MATLAB) }\end{array}$ \\
\hline
\end{tabular}

fault locations between $3-35$ and between $2-4$ have no alternative paths from the generators. The ATGs again ramp up to their maximum power to service the priority loads; nonvital loads at nodes 2, 3, 8, and 10 are shed, while the nonvital load at node 1 is partially serviced due to insufficient power from the ATG node 11. However, all the vital and the semi-vital loads are fully serviced. In the global optimum solution shown in Fig. 5, $S W 3, S W 35, S W 5$, and $S W 810$ are open and $S W 4, S W 46$, $S W 6$, and $S W 79$ are closed. Table III shows that the global optimum solution for $P 3$ produces the same switch configuration as the global optimum solution for $P 1$. Also, the total power delivered to the loads is almost the same in both cases. The local optimum solution for $P 2$ is, however, different in one switch position ( $S W 2$ closed instead of $S W 1$ ) and marginally less total power is delivered to the loads. Further extensive simulations are performed with various fault scenarios to compare solutions provided by $P 1, P 2$, and $P 3$. No significant difference in the total power delivered is observed after reconfiguration, even though switch status for the three solutions may differ for a small number of cases.

Next, an analytical study of the robustness of the system is provided using the model in Fig. 2. CDF of the power delivered to the loads is used to analyze the robustness against several random faults and is defined as 


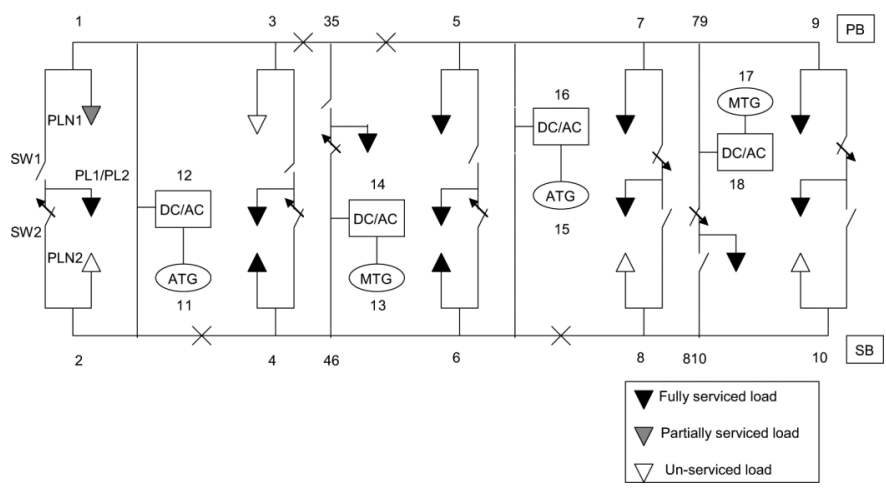

Fig. 5. Schematic view of SPS under faults occurring at $3-35,35-5,2-4$, and $6-8$.

TABLE III

POWER DELIVERED TO LOADS UNDER FAULTS OCCURRING AT $3-35,35-5,2-4$, AND $6-8$

\begin{tabular}{|c|c|c|c|c|c|c|c|}
\hline $\begin{array}{l}\text { Load } \\
\text { positions }\end{array}$ & $\begin{array}{l}\text { Vital } \\
\text { (MW) }\end{array}$ & $\begin{array}{l}\text { Semi- } \\
\text { vital } \\
(\mathrm{MW})\end{array}$ & $\begin{array}{l}\text { Non- } \\
\text { vital } \\
\text { (MW) }\end{array}$ & $\begin{array}{l}\text { SW } \\
\text { positions } \\
\text { (closed) }\end{array}$ & $\begin{array}{l}\text { MTG } \\
\text { (Total) } \\
\text { (MW) }\end{array}$ & $\begin{array}{l}\text { ATG } \\
\text { (Total) } \\
\text { (MW) }\end{array}$ & Solver \\
\hline $\begin{array}{l}\text { Load } 1 / 2 \\
\text { Load 3/4 } \\
\text { Load } 35 \\
\text { Load 5/6 } \\
\text { Load } 7 / 8 \\
\text { Load } 810 \\
\text { Load 9/10 }\end{array}$ & $\begin{array}{l}0.5000 \\
0.5000 \\
0.5000 \\
0.5000 \\
0.5000 \\
0.5000 \\
0.5000\end{array}$ & $\begin{array}{l}1.0000 \\
1.0000 \\
1.0000 \\
1.0000 \\
1.0000\end{array}$ & $\begin{array}{l}0.4786 \\
0.5000 \\
1.0000 \\
0.5000 \\
0.5000\end{array}$ & $\begin{array}{l}\text { SW2 } \\
\text { SW4 } \\
\text { SW46 } \\
\text { SW6 } \\
\text { SW7 } \\
\text { SW79 } \\
\text { SW9 }\end{array}$ & 8.5949 & 4.0000 & $\begin{array}{l}\text { Global } \\
\text { Non-convex with } \\
\text { mixed integer } \\
\text { (LINGO) }\end{array}$ \\
\hline $\begin{array}{l}\text { Load } 1 / 2 \\
\text { Load 3/4 } \\
\text { Load } 35 \\
\text { Load 5/6 } \\
\text { Load } 7 / 8 \\
\text { Load } 810 \\
\text { Load } 9 / 10\end{array}$ & $\begin{array}{l}0.5000 \\
0.5000 \\
0.5000 \\
0.5000 \\
0.5000 \\
0.5000 \\
0.5000\end{array}$ & $\begin{array}{l}1.0000 \\
1.0000 \\
1.0000 \\
1.0000 \\
1.0000\end{array}$ & $\begin{array}{l}0.3212 \\
0.6506 \\
1.0000 \\
0.5000 \\
0.5000\end{array}$ & $\begin{array}{l}\text { SW1 } \\
\text { SW4 } \\
\text { SW46 } \\
\text { SW6 } \\
\text { SW7 } \\
\text { SW79 } \\
\text { SW9 }\end{array}$ & 8.6131 & 4.0000 & $\begin{array}{l}\text { Local } \\
\text { Non-convex with } \\
\text { integer relaxation } \\
\text { (MATLAB) }\end{array}$ \\
\hline $\begin{array}{l}\text { Load } 1 / 2 \\
\text { Load } 3 / 4 \\
\text { Load } 35 \\
\text { Load } 5 / 6 \\
\text { Load } 7 / 8 \\
\text { Load } 810 \\
\text { Load } 9 / 10\end{array}$ & $\begin{array}{l}0.5000 \\
0.5000 \\
0.5000 \\
0.5000 \\
0.5000 \\
0.5000 \\
0.5000\end{array}$ & $\begin{array}{l}1.0000 \\
1.0000 \\
1.0000 \\
1.0000 \\
1.0000\end{array}$ & $\begin{array}{l}0.3421 \\
0.6364 \\
1.0000 \\
0.5000 \\
\\
0.5000\end{array}$ & $\begin{array}{l}\text { SW2 } \\
\text { SW4 } \\
\text { SW46 } \\
\text { SW6 } \\
\text { SW7 } \\
\text { SW79 } \\
\text { SW9 }\end{array}$ & 8.6004 & 4.0000 & $\begin{array}{l}\text { Global } \\
\text { Convex with } \\
\text { integer relaxation } \\
\text { (MATLAB) }\end{array}$ \\
\hline
\end{tabular}

$C D F=$ Prob. $\left\{\right.$ Power delivered to loads $\left.\leq P_{D}\right\}$, where $P_{D}$ is the desired power. As shown in Fig. 7, the CDF of the power delivered to the loads is plotted for all possible random locations for two, three, and four faults. The pre-fault configuration for this system is shown in Fig. 6. There are several observations that can be drawn from the CDF analysis of the system which are summarized in the following.

1) The total load that are serviced is $17.5 \mathrm{MW}$ of which the total power required to serve the vital loads is $3.5 \mathrm{MW}$, total power required for the serving the semi-vital loads is 7 $\mathrm{MW}$, and the rest $7 \mathrm{MW}$ is used to serve the nonvital loads. Table IV shows the percentage of the total load served for various random fault cases.

2) For all possible 2-fault cases, the system is able to sustain power delivered to about 16.47MWin $50 \%$ ofthecases.Forrandom 3 faultcases, thepowerdelivereddipsto 15.16

MW whereas for random 4-fault cases, the power delivered to loads further reduces to $14 \mathrm{MW} 50 \%$ of the cases. Therefore, Fig. 7 and Table IV confirm that $100 \%$ of the

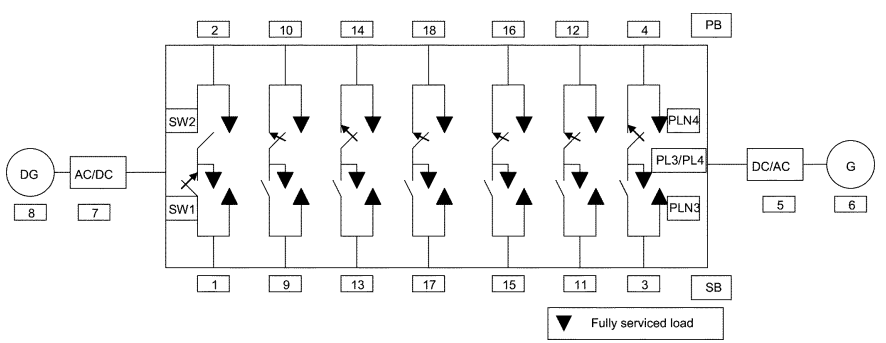

Fig. 6. Schematic view of SPS under normal condition (pre-fault).

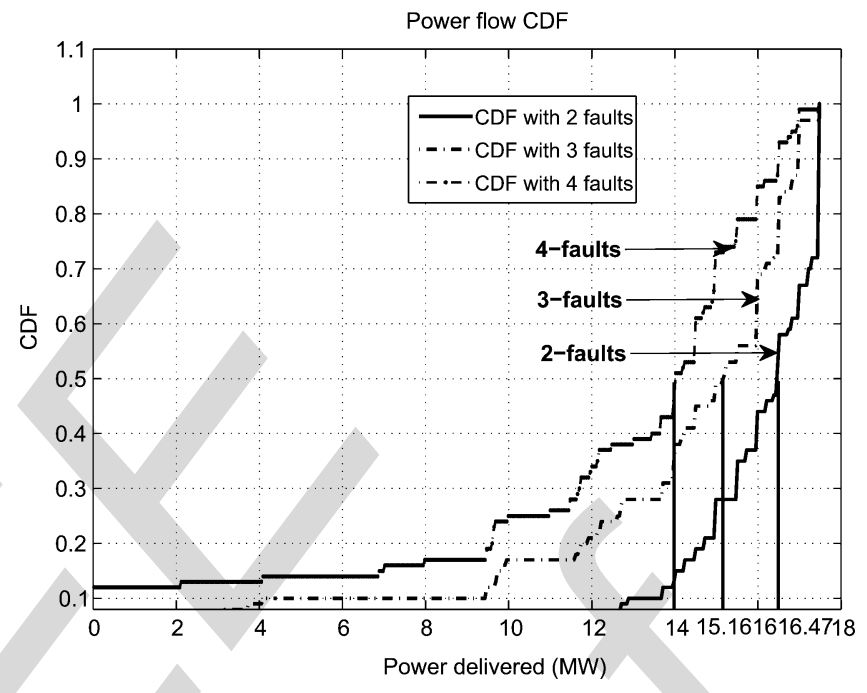

Fig. 7. CDF of power delivered (MW).

TABLE IV

POWER Delivered as a Function of Faults

\begin{tabular}{|c|c|c|c|}
\hline Percentage & 2 -faults & 3-faults & 4 -faults \\
\hline 50 & $16.47 \mathrm{MW}$ & $15.16 \mathrm{MW}$ & $14 \mathrm{MW}$ \\
\hline 10 & $13.2 \mathrm{MW}$ & $4.09 \mathrm{MW}$ & $0 \mathrm{MW}$ \\
\hline
\end{tabular}

vital loads are served for these fault cases $50 \%$ of the time, and $100 \%$ of the semi-vital loads are served $50 \%$ of the time. The nonvital loads are, however, shed to maintain power delivered to the higher priority loads.

3) For random 2-fault cases, the system is able to serve $100 \%$ of the vital loads and $100 \%$ of the semi-vital loads for $90 \%$ of the cases. For random 3-fault cases, vital loads are not served only about $10 \%$ of the time, while for 4 -fault cases, vital loads are not served $15 \%$ of the time. These are cases where faults occur at the generator buses.

Therefore, the CDF tool based robustness study of the shipboard power system reconfiguration incorporates a probabilistic view of the robustness of the system to various random faults.

Finally, the results of the dual-objective formulation in (22) of the optimization problem is presented. The weight $w$ (22) is varied from 0 to 1 to investigate the combined objective of the power delivered to the loads $\left(F_{1}\right)$ and the number of switching operations $\left(F_{2}\right)$. In Figs. 8 and 9, a case with two faults occurring between $12-4$ and $7-1$ with model in Fig. 6 is presented which shows power delivered to the loads and number of switch operations performed, respectively. As the weight $w$ is increased from 0 to 1, Fig. 9 shows an increase in 


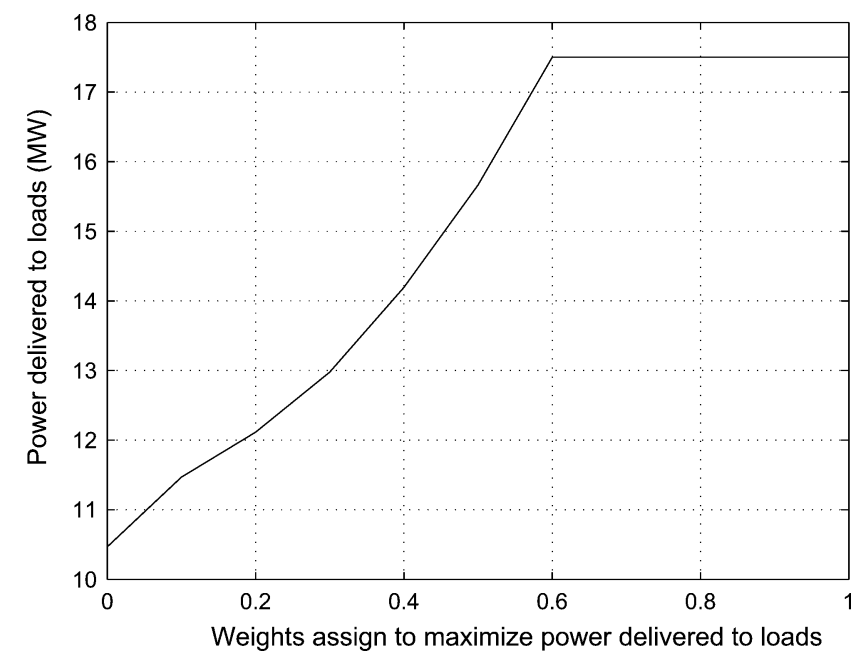

Fig. 8. Power delivered for faults between $12-4$ and $7-1$.

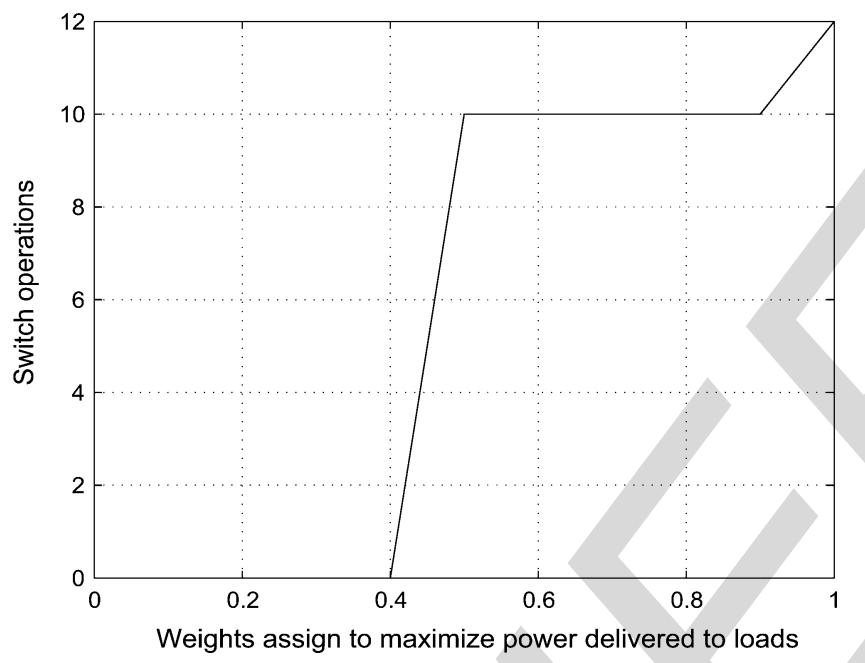

Fig. 9. Number of switching performed for faults between $12-4$ and $7-1$.

the number of switch operations. When $w$ increases from 0.4 to 0.5 , the number of switch pair operations increases from 0 to 5 . This is due to the fact that the switching surface allows more number of switches to change suddenly and accounts for a more stable locally optimal state. With the weighted objective function, when relatively more importance is given to power delivery, the number of switch operations also increases, which is expected as more switching is needed to deliver more power to the loads. As seen in Figs. 8 and 9, more than five switch pair changes, however, do not increase the amount of power delivered to the loads in this particular example. Therefore, five switch pair changes are sufficient to deliver maximum power to the loads, corresponding to the best trade-off between the power delivered and number of switching operations.

In a separate analysis, a step-by-step study of the reconfiguration solution with the assumption that only one pair of switches is allowed to change at each step of reconfiguration is shown in Table V. The first column indicates the initial switch state; final state when the reconfiguration is complete is shown in the last column, and all the other columns in between represent the intermediate switch states resulting in maximum incremental
TABLE V

POWER DELIVERED AND SWITCH TRANSITION TO FAULTS OCCURRING AT $2-10$ AND $12-4$

\begin{tabular}{|c|c|c|c|c|c|c|}
\hline $\begin{array}{c}\text { Power delivered } \\
\text { to loads }\end{array}$ & 12.3652 & 13.8652 & 15.3653 & 16.8653 & 17.1503 & 17.5000 \\
\hline SW1 & 1 & 1 & 1 & 1 & 1 & 1 \\
SW2 & 0 & 0 & 0 & 0 & 0 & 0 \\
SW3 & 0 & 0 & 0 & 0 & 0 & 1 \\
SW4 & 1 & 1 & 1 & 1 & 1 & 0 \\
SW9 & 1 & 1 & 1 & 1 & 0 & 0 \\
SW10 & 0 & 0 & 0 & 0 & 1 & 1 \\
SW11 & 1 & 1 & 1 & 1 & 1 & 1 \\
SW12 & 0 & 0 & 0 & 0 & 0 & 0 \\
SW13 & 0 & 0 & 0 & 1 & 1 & 1 \\
SW14 & 1 & 1 & 1 & 0 & 0 & 0 \\
SW15 & 0 & 1 & 1 & 1 & 1 & 1 \\
SW16 & 1 & 0 & 0 & 0 & 0 & 0 \\
SW17 & 0 & 0 & 1 & 1 & 1 & 1 \\
SW18 & 1 & 1 & 0 & 0 & 0 & 0 \\
\hline
\end{tabular}

power delivery to loads from a previous column. Each switch state deliver power to loads incrementally from 12.36 MW to 17.5 MW. The analysis is particularly useful when cost associated with switch operations becomes significant; thereby only a limited number of switch operations have to be performed in restoring power to the loads after reconfiguration. Obviously, the solution may not be globally optimal when the number of switch pair changes is restricted to less than 5, but will ensure maximum power delivery for a fixed number of switch operations.

\section{COMputational COMPleXity}

In this section, first, the complexity of the solutions provided to optimally reconfigure the SPS is analyzed. "LINGO" software is used to solve the original nonconvex mixed integer problem. It uses "branch-and-bound" method to maintain a provable upper and lower bound on the objective value which is $\epsilon$-suboptimal to the global optimum. First, assuming convexity, the algorithm finds an upper and a lower bound on the optimal objective value $p^{*}$. If the difference between any upper and lower bounds satisfies $U_{k}-L_{k} \leq \epsilon$, it terminates or, otherwise, creates branches for any index $k \in 1, \ldots n$ on the switch variables to form two subproblems. Using convex relaxations on the switches other than the $k$ th switch, it produces and upper and lower bound on the optimal value of each subproblem. The optimal value $p^{*}$ of the original problem is the smaller of the two subproblems. This eventually forms a binary tree with each leaf node created by fixing a variable that is not fixed in the parent node. So, a node at depth $i$ in the tree corresponds to a subproblem with $i$ switch variables have fixed values. The upper and lower bound on $p^{*}$ are obtained by the minimum of upper and lower bounds over all the leaf nodes. The algorithm terminates when $U_{i}-L_{i} \leq \epsilon$. Therefore, in the worst case, a complete binary tree is developed to depth $n$ which makes the complexity as $2^{n}$ where $n$ is the number of switch variables. For our problem with $n=14$, the worst-case complexity is $2^{14}$ which is relatively large for this reconfiguration problem. The worst-case complexity for interior-point based method is $\mathrm{O}\left(n m^{2}\right)$, where $n$ is the number of variables, $m$ is the number of constraints, and $n>m$. The complexity of this method is thus polynomial in time but in worst-case much less that the "branch-and-bound" based method. The Interior-point method solves the reconfiguration problem by applying Newton's 
TABLE VI

EXECUTION TIME EFFICIENCY (ETE) FOR ORIGINAL NONCONVEX AND TRANSFORMED CONVEX METHODS. REFERENCE EXECUTION TIME FOR ORIGINAL NONCONVEX METHOD $=1 \mathrm{~s}$

\begin{tabular}{|c|c|c|}
\hline Number of variables $(n)$ & Constraints $(m)$ & ETE \\
\hline 8 & 6 & 1.125 \\
\hline 14 & 12 & 0.123 \\
\hline 30 & 28 & 0.002 \\
\hline
\end{tabular}

method to a sequence of equality constrained problems. Results show that local optimum found by interior-point based solvers match global optima by "branch-and-bound" method for this reconfiguration problem. This is due to the fact that the problem is highly convex with some nonconvexity in the generator constraints. Our convex formulation of the relaxed-integer nonconvex problem reinforce this claim. The complexity of this relaxed-integer convex problem is, however, the same as the relaxed-integer nonconvex problem although a global optimum of this problem can be found for the former as any local optimum for a convex problem is the global optimum solution. In this case, sequential convex programming [18] is applied by maintaining estimate of solution $x^{(k)}$ and a trust region $T^{(k)} \subset R^{n}$ such that (18) is affine. With relaxed-integer convex formulation, we are able to convincingly state that our reduced-complexity solution provides exactly the same result as the "LINGO" based global optimum solution presented in [14]. In Table VI, the ETE-percentage efficiency in execution - is defined as

$$
E T E=\frac{\text { Execution time for transformed convex }}{\text { Execution time for original nonconvex. }}
$$

This shows that assuming platform independence, if the execution time for original nonconvex method with $n=14$ and $m=12$ is $1 \mathrm{~s}$, the proposed convex method will provide the same result in $123 \mathrm{~ms}$. For low values of $n$ and $m$, exhaustive search is easy to perform. Therefore, there is no gain in transforming the nonconvex problem. As Table VI shows, for $n=8$ and $m=6$, branch and bound performs better. As the number of switches increases, a very high execution time efficiency is possible.

\section{CONCLUSION}

In this paper, two centralized optimization solutions are evaluated that deliver near-optimal power to loads in shipboard power system. The complexity of proposed solutions is polynomial in time and much lower than the complexity of the global solver that uses "branch and bound". Local optimum for relaxed-integer nonconvex formulation and global optimum for relaxed integer convex formulation match the global optimum for original MINLP nonconvex formulation with high regularity. Further, cumulative distribution of power flow is used to show that in $50 \%$ of the fault cases (up to four random faults), the vital and semi-vital loads are serviced. Analysis of the tradeoff between power delivery and number of switching operations performed during reconfiguration is also provided. As expected, it is observed that an increase in switching is necessary to deliver more power to loads. Furthermore, change of switch states is tracked from pre-fault state to final state, while 1) changing one switch pair at a time and 2) maximizing power delivery at each state. The tradeoff between number of switch operations and power delivered is also quantified.

\section{REFERENCES}

[1] K. N. Miu, H.-D. Chiang, B. Yuan, and G. Darling, "Fast service restoration for large-scale distribution systems with priority customers and constraints," in Proc. 20th Int. Conf. Power Industry Computer Applications, 1997, vol. 13, pp. 789-795.

[2] E. R. Ramos, J. L. Martinez-Ramos, A. G. Exposito, and A. J. U. Salado, "Optimal reconfiguration of distribution networks for power loss reduction," in Proc. IEEE Porto Power Tech, 2001, vol. 3, p. 5.

[3] A. N. Guimaraes, J. F. C. Lorenzeti, and C. A. Castro, "Reconfiguration of distribution systems for stability margin enhancement using tabu search," in Proc. Int. Conf. Power System Technology, 2004, vol. 2, pp. 1556-1561.

[4] A. L. Morelato and A. J. Monticelli, "Heuristic search approach to distribution system restoration," IEEE Trans. Power Del., vol. 4, no. 4, pp. 2235-2241, Oct. 1989.

[5] C. S. Chen, C. H. Lin, and H. Y. Tsai, "A rule-based expert system with colored petri net models for distribution system service restoration," IEEE Trans. Power Syst., vol. 17, no. 4, pp. 1073-1080, Nov. 2002.

[6] T. Nagata and H. Sasaki, "An efficient algorithm for distribution network restoration," in Proc. IEEE Power Eng. Soc. Summer Meeting, 2001, vol. 1, pp. 54-59.

[7] Y. Hsiao and C. Chien, "Enhancement of restoration service in distribution systems using a combination fuzzy-GA method," IEEE Trans. Power Syst., vol. 15, no. 4, pp. 1394-1400, Nov. 2000.

[8] K. L. Butler, N. D. R. Sarma, and V. R. Prasad, "A new method of network reconfiguration for service restoration in shipboard power systems," in Proc. IEEE PES Transmission and Distribution Conf., 1999, vol. 2, pp. 658-662.

[9] K. L. Butler, N. D. R. Sarma, and V. R. Prasad, "Network reconfiguration for service restoration in shipboard power distribution systems," IEEE Trans. Power Syst., vol. 16, no. 4, pp. 653-661, Nov. 2001.

[10] K. L. Butler-Purry, "Multi-agent technology for self-healing shipboard power systems," in Proc. 13th Int. Conf. Intelligent Systems Application to Power Systems, 2005, p. 5.

[11] K. L. Butler-Purry and N. D. R. Sarma, "Self-healing reconfiguration for restoration of naval shipboard power systems," IEEE Trans. Power Syst., vol. 19, no. 2, pp. 754-762, May 2004.

[12] J. Billo, "Models and methods for shipboard power system reconfiguration," Master's thesis, Univ. Texas, Austin, 2003.

[13] S. Khushalani and N. N. Schulz, "Restoration optimization with distributed generation considering islanding," in Proc. IEEE Power Eng. Soc. General Meeting, 2005, vol. 3, pp. 2445-2449.

[14] S. Khushalani, J. Solanki, and N. N. Schulz, "Optimized restoration of combined ac/dc shipboard power systems including distributed generation and islanding techniques," Elect. Power Syst. Res., vol. 78, pp. 1528-1536, 2008.

[15] V. Balakrishnan, S. Boyd, and S. Balemi, "Branch and bound algorithm for computing the minimum stability degree of parameter-dependent linear systems," Int. J. Robust Nonlin. Control, vol. 1, no. 4, pp. 295-317, Oct.-Dec. 1991.

[16] S. Bose, S. Pal, C. Scoglio, B. Natarajan, S. Das, and N. N. Schulz, "Analysis of optimized reconfiguration of power systems for electric ships," in Proc. North American Power Symp., Sep. 26-28, 2010, pp. $1-7$.

[17] S. Bose, B. Natarajan, C. Scoglio, S. Das, and N. N. Schulz, "Analysis of robustness for shipboard power system with non-radial power flow," in Proc. IEEE Electric Ship Technologies Symp., Apr. 26-28, 2011, pp. $1-7$.

[18] S. Boyd and L. Vandenberghe, Convex Optimization. Cambridge, U.K.: Cambridge Univ. Press, 2004.

[19] P. R. Chester and W. R. Jay, "Zonal electrical distribution systems: An affordable architecture for future," Naval Eng. J., pp. 45-51, 1993.

[20] J. D. Glover, M. S. Sarma, and T. J. Overbye, Power System Analysis and Design, 4th ed. Tampa, FL: Thomson, 2008.

[21] L. Akter and B. Natarajan, "QoS constrained resource allocation to secondary users in cognitive radio networks," Comput. Commun., vol. 32, pp. 1923-1930, 2009.

[22] T. Marler, "A study of multiobjective optimization methods for engineering application,” Ph.D. dissertation, Univ. Iowa, Iowa City, 2005. 
Sayak Bose (S'10) received the B.Tech. degree from Kalyani University, India, in 2003 and the M.S. degree from the University of Kansas, Lawrence, in 2009. Currently, he is pursuing the Ph.D. degree from Kansas State University, Manhattan.

He worked as an Assistant Systems Engineer at Tata Consultancy Services, India, for over three years. His research interests are communication theory and systems, optimized reconfiguration analysis, sensor placement, and network control.

Siddharth Pal is pursuing the B.Tech. degree in the Electronics and Telecommunication Department, Jadavpur University, Kolkata, India.

He spent the summer of 2010 as an undergraduate researcher at Kansas State University, Manhattan. His research interests include evolutionary computation, multi-objective optimization, image processing, robotics, and engineering application of AI.

Balasubramaniam (Bala) Natarajan (SM'08) received the Ph.D. degree from Colorado State University, Fort Collins, in 2002.

$\mathrm{He}$ is an Associate Professor in the Department of Electrical and Computer Engineering at Kansas State University, Manhattan. His main research interests include communications theory; wireless systems (physical layer) and spread spectrum; multi-carrier modulation and diversity combining; channel modeling; fiber optic communications; and power system reconfiguration.
Sanjoy Das received the B.S. degree in electrical engineering from Sambalpur University, Orissa, India, in 1987 and the M.S. and Ph.D. degrees in electrical engineering from Louisiana State University, Baton Rouge, in 1994.

Between 1994 and 1997, he received his postdoctoral training from the University of California, Berkeley, and the Smith-Kettlewell Institute, San Francisco, CA. He has also worked as a Research Scientist at ITT systems. Since 2001, he has been in the Electrical and Computer Engineering Department at Kansas State University, Manhattan, where he is currently an Associate Professor. His research interests include evolutionary algorithms, swarm intelligence, artificial immune systems, multiobjective optimization, neural networks, intelligent agents, and computational intelligence.

Noel N. Schulz (SM'00) received the B.S.E.E. and M.S.E.E. degrees from Virginia Polytechnic Institute and State University (Virginia Tech), Blacksburg, in 1988 and 1990, respectively, and the Ph.D. degree in electrical engineering from the University of Minnesota, Minneapolis, in 1995.

She is the Paslay Professor of Electrical and Computer Engineering at Kansas State University, Manhattan. She has served as a faculty member at Mississippi State University and Michigan Tech in the past. Her research interests are in computer applications in power system operation including artificial intelligence techniques.

Dr. Schulz has been active in the IEEE Power and Energy Society, currently serving as President-Elect. She is recipient of the IEEE/PES Walter Fee Outstanding Young Power Engineer Award and NSF CAREER award. She is a member of ASEE, SWE, Eta Kappa Nu, and Tau Beta Pi.

Caterina M. Scoglio (M'04) received the Dr. Eng. degree from the "Sapienza" Rome University, Rome, Italy, in 1987.

She is an Associate Professor in the Department of Electrical and Computer Engineering at Kansas State University, Manhattan, since 2005. Her main research interests include the modeling and analysis of complex networks, with applications in epidemic spreading and power grids. Before joining Kansas State University, she worked at the Fondazione Ugo Bordoni from 1987 to 2000, and at the Georgia Institute of Technology, Atlanta, from 2000 to 2005. 